\title{
LABORATORY SIMULATION OF LOW EARTH ORBIT (LEO) ATOMIC OXYGEN EFFECTS*
}

\author{
George E. Caledonia, Robert H. Krech and David B. Oakes \\ Physical Sciences Inc.
}

\section{ABSTRACT}

PSI's pulsed fast oxygen atom source has been used extensively over the last 7 years to investigate the effects of ambient oxygen atoms impacting materials placed in LEO. During this period we have irradiated well over 2000 material samples with $8 \mathrm{~km} / \mathrm{s}$ oxygen atoms generated in our source. Typical irradiance level is $3 \times 10^{20} \mathrm{O}$ atoms $/ \mathrm{cm}^{2}$ although some materials have been irradiated to fluence levels as high as $6 \times 10^{21} \mathrm{O}$ atoms $/ \mathrm{cm}^{2}$.

We will review the operating principles and characteristics of our source along with the diagnostic and handling procedures appropriate to material testing. We will then present representative data on the velocity dependence of oxygen atom erosion rates (the PSI source provides oxygen atoms tunable over the velocity range of 5 to $12 \mathrm{~km} / \mathrm{s}$ ) as well as the dependence on material temperature. Specific examples of non-linear oxidative effects related to surface contamination and test duration will also be provided.

\section{INTRODUCTION}

We developed a pulsed fast oxygen atom beam in the mid $1980^{\prime} \mathrm{s}^{\prime}$ under NASA funding to provide a ground test facility to qualify materials to be used in low earth orbit (LEO). This was in response to a number of early space shuttle observations ${ }^{2.5}$ of material erosion and property change resulting from interactions with the LEO ambient atmosphere, which is dominantly atomic oxygen. These interactions occur at orbital velocities, $-8 \mathrm{~km} / \mathrm{s}$, and for a while there was a frenzied activity to develop atomic oxygen beams which operate at this velocity. The manifold of developed ground sources has been reviewed previously and to our knowledge ours is the only system that provides a high flux neutral oxygen atom beam with wide area operating capability at the desired velocity of $8 \mathrm{~km} / \mathrm{s}$. Sets of materials ( $2.5 \times 2.5 \mathrm{~cm}$ samples) have been simultaneously irradiated to $8 \mathrm{~km} / \mathrm{s}$ oxygen atom fluences of $6 \times 10^{21} \mathrm{~cm}^{-2}$ in our facility.

Simultaneous with the development of ground test facilities, a number of flight programs dedicated to evaluating the effect of the LEO environment on materials were also configured and flown. Notable examples of these include the Long Duration Exposure Facility ${ }^{7.9}$ and the Evaluation of Oxygen Interactions Shuttle Experiments, ${ }^{10-11}$ the most recent of which, EOIM-3, flew in August 1992.

A large data base on LEO environment material effects has been developed as a result of these flight and ground testing activities. Some of this data base is scattered and much can be difficult to interpret due, for example, to contaminant effects on flight tests and the lack of characterization of ground facilities.

Flight tests of materials are preferable but considerably more expensive than ground evaluation. Furthermore, it is difficult to acquire material aging data from flight tests which, LDEF notwithstanding, are generally limited to less than 2 weeks experiment duration. Lastly, the flight data represent an average over the orbital environmental conditions of the flight. Many materials exhibit an increased rate of oxidative behavior with increased temperature, e.g. Ref. 12. Materials can vary in temperatures between $-40^{\circ} \mathrm{C}$ to $+65^{\circ} \mathrm{C}$ as the surface exposure varies from deep space to solar illumination (pathological materials excluded). Thus a measurement over a short shuttle experiment may not be reliably extended to a longer mission with different orbits, even if the aging effects can be linearly extrapolated.

The use of ground test data has been problematical both because facility definitions have been vague and because standardized measurement techniques have not been applied. The need for a measurement protocol is clear. Banks et al. have recently discussed this for asher systems ${ }^{13}$ (a technique which has seen some abuse in the past, ${ }^{14}$ ) and Minton ${ }^{15}$ has recently

"This work was supported in part by the Defense Nuclear Agency, monitored by Dr. William Blumberg of the Geophysics Directorate of the USAF Phillips Laboratory, and by the NASA Lyndon B. Johnson Space Center, monitored by Mr. Steve Fitzgerald. 
presented version 1 of a detailed protocol. NASA Langley has responsibility under the SEE program to ultimately provide a definitive protocol.

PSI has performed commercial evaluation of LEO materials since 1987 and has developed a measurement protocol very similar to that outlined by Minton. The first step in such a protocol is the clear definition of the capabilities and operating properties of the fast oxygen atom facility. Since the PSI O-atom beam source has been used for material evaluation for the past 7 years, a significant effort has been dedicated to characterizing its operating properties in detail.

The operating principles and characteristics of our device are described below. This discussion is followed by some representative results of material behavior under simulated LEO conditions.

\section{The Fast Atom Source}

The hyperthermal oxygen atom source and experimental configuration have been described previously ${ }^{16,17}$ and will be discussed only briefly here. A schematic of the experimental system is shown in Figure 1 . It is comprised of two stainless steel six-way crosses, including an 8 in. cross source chamber housing a pulsed oxygen valve/nozzle assembly, connected to a 16 in. cross expansion chamber. The system is pumped by a cryopump attached to the large cross which maintains a base pressure of $3 \times 10^{-7}$ Torr.

The oxygen atoms are generated in a pulsed laser discharge of pure $\mathrm{O}_{2}$ as described by Caledonia et al.' A 12-J/pulse $\mathrm{CO}_{2}$ laser is focused with a $100 \mathrm{~cm}$ focal length $\mathrm{BaF}_{2}$ lens into the throat of a 20 -deg full angle, $12.5 \mathrm{~cm}$ long conical expansion nozzle which has been partially filled with $\mathrm{O}_{2}$ by a pulsed beam valve. The plasma, ignited at the throat, expands out the nozzle dissociating the molecular oxygen in front of it. The nozzle was designed to allow ion and electron recombination while the slower kinetics of atom/atom recombination maintains a highly dissociated beam. The result, at $8 \mathrm{~km} / \mathrm{s}$, is an approximately $50 \mu s$ pulse of highly dissociated oxygen ( $>80 \%$ atoms) with less than $1 \%$ ion content (the beam is charge neutral). The general beam properties are provided in Table 1 while the diagnostics used in evaluating these properties are summarized in Table 2.

The beam velocity ( 5 to $12 \mathrm{~km} / \mathrm{s}$ ) is selected by varying the time delay between the pulsing of the $\mathrm{O}_{2}$ valve and the triggering of the $\mathrm{CO}_{2}$ laser. The delay determines the mass of $\mathrm{O}_{2}$ processed by the pulsed discharge which is inversely related to the beam velocity. The velocity is determined by measuring the time of flight of the pulse between two radiometers attached to the source chamber, mounted along the beam axis and separated by $7.6 \mathrm{~cm}$, and filtered to monitor the ${ }^{5} \mathrm{~S}-\mathrm{S} P$ oxygen atom line $(777$ nm) (see Figure 1).

We have estimated the flux of oxygen atoms generated per pulse by measuring the mass of $\mathrm{O}_{2}$ processed per pulse. This was determined by using the known time delay between the pulsing of the oxygen valve and the firing of the laser, monitoring the flow of oxygen with a mass flow meter and measuring the temporal profile of the molecular oxygen pulse (laser off) with a pressure transducer aligned along the beam axis. With this measure of the $\mathrm{O}_{2}$ processed per pulse, the flux at any point down stream can be estimated if the expansion characteristics of the beam are known. The expansion properties have been determined previously ${ }^{13.17}$ by measuring the fast $\mathrm{O}$-atom erosive mass loss of polyethylene samples mounted $75 \mathrm{~cm}$ downstream from the nozzle throat as a function of radial position. The expansion half angle (to le times the on-axis flux) was found to be $14 \mathrm{deg}$. These calibrations have been cross checked by measuring the absolute oxidation rates of materials and comparing them with equivalent space flight measurements. More recently, as discussed in the next section, the oxidation rate of thin silver films has been used as a calibration standard.

Although the beam is charge neutral, it does have a small but finite ion content. The charge concentration can be decreased by up to a factor of eight using a pair of pseudo-Helmholtz coils as shown in Figure 2 . Thus any effects due to charged rather than neutral species can be delineated. Control of the ionic concentration is critical in that some materials are efficiently oxidized by $\mathrm{O}^{+}$and not by $\mathrm{O}$. The local charge concentration can be measured through use of a retractable Langmuir probe, Figure 2. The probe is positionable both horizontally and vertically and it has been found that the spatial and temporal shape of the ion distribution matches that of the neutral distribution. ${ }^{17}$

We have recently measured the $O(' D)$ concentration of the beam by monitoring the emission from the forbidden $O\left({ }^{\prime} D \rightarrow{ }^{3} P\right)$ transition using a specially constructed bank of narrow-band interference filters. The $O(' D)$ concentration was found to be $s 0.4 \%$ for a beam velocity of $8 \mathrm{~km} / \mathrm{s}$ and to increase monotonically with beam velocity over the velocity range of 6 to $12 \mathrm{~km} / \mathrm{s}$. 
We have also evaluated the translational temperature of the beam. This was done by passing the O-beam through a $0.15 \mathrm{~cm}$ diameter circular aperture and measuring the oxidation pattern on a thin silver film witness plate placed $5 \mathrm{~cm}$ behind the aperture. Any growth in the beam beyond the slit width is due to the Maxwellian motion of the $O$ atoms and can be related to an effective temperature for an $8 \mathrm{~km} / \mathrm{s}$ velocity beam. This temperature was deduced by be $-300 \mathrm{~K}$ at a distance of $85 \mathrm{~cm}$ from the nozzle throat. The beam temperature will be higher closer to the throat and is calculated to be $\sim 1000 \mathrm{~K}$ at the nozzle exit plane.

Lastly, we have measured the VUV/UV content of the beam and find that it is somewhat less then the equivalent LEO value per oxygen atom. Since the beam is pulsed, the VUV radiation can be chopped allowing independent examination of VUV effects on materials.

Our O-beam facility has been employed on several occasions to pre-test materials which were subsequently flown in space experiments. In general, the comparison between flight data and ground test data has been quite good. The most recent large ground test was for the SEE program on materials for the EOIM-3 flight. ${ }^{18}$ We irradiated 84 materials to a fluence of $2 \times 10^{20} \mathrm{~cm}^{-2} 8 \mathrm{~km} / \mathrm{s}$ oxygen atoms and followed a careful protocol of pre- and post-test weighing taking account of water uptake. The Jet Propulsion Laboratory performed subsequent surface analysis. With a few understood exceptions, the comparison between our ground test results for these materials and the flight observations was quite good, ${ }^{18}$ demonstrating our ability to simulate LEO conditions.

\section{EXAMPLE MATERIAL STUDIES}

Material oxidation rates can be significantly affected both by the test environment and the test system parameters. In this section we will briefly examine the effect of sample temperature and o-atom velocity on two materials, a thin silver film and $\mu$-crystalline carbon. In the case of the thin silver film the effect of two additional parameters, test duration and surface contamination will be addressed.

We have performed investigations of the temperature dependent oxidative behavior of several materials over the temperature range of 0 to $85^{\circ} \mathrm{C}$. An example of such studies is our observations of the oxidative behavior of thin films of silver $(600 \AA)^{19}$ and $\mu$-crystalline carbon $(1 \mu \mathrm{m})$ which were vacuum deposited onto the crystals of quartz crystal monitors (QCM's). QCM's are the diagnostic of choice for monitoring mass change as the measurements are performed continuously in-situ. Unfortunately in many instances the materials to be tested cannot be coated on a crystal. Then weight change is monitored by preand post-testing weighing using our Mettler analytical balance and Cahn micro-balance. (Here a careful protocol must be followed to account for moisture uptake).

The silver and carbon films are good examples for consideration in that the silver gains mass upon oxidation while the carbon loses mass. In these experiments, the $\mathrm{QCM}$ was mounted directly onto a temperature-controlled aluminum block and thus the quartz crystal, which is in intimate thermal contact with the $Q C M$ base, was maintained to $\pm 0.5^{\circ} \mathrm{C}$ (material coupons can be monitored in the same manner). Temperature regulation of the QCM was necessary since the oscillator frequency is temperature dependent. Therefore, a change in temperature could be erroneously interpreted as a mass gain or loss.

The silver films are particularly interesting since because of their high oxidation efficiency we feel that they can be used as a standard for laboratory flux calibrations. Furthermore, they have been successfully used in both flight ${ }^{19}$ and laboratory ${ }^{17}$ studies to study the distributions or hyperthermal oxygen atoms scattered off of selected surfaces. The oxidation behavior of these films exhibits several intriguing effects. Our studies in this are described in detail in Ref. 20 and presented briefly below.

Typical thin film silver oxidation histories for irradiation by $8 \mathrm{~km} / \mathrm{s}$ oxygen atoms at room temperature are shown in Figure 3. Shown is oxidation depth versus the number of impinging 0 -atom pulses as determined by the measured mass increase for two film thicknesses. Two important features can be seen. First, no oxidation occurs during the first two hundred pulses. This is typically observed and we relate it to the required removal of a contamination layer on the silver film. Indeed, if we first irradiate the film with several hundred pulses of a fast argon beam and then shortly thereafter impinge it with fast oxygen atoms, we find that oxidation initiation is immediate. Second, after oxidation initiation, the oxidation depth increases linearly with $\mathrm{O}$-atom fluence for approximately the first $250 \AA$ of the silver film and then the oxidation rate slows down. We believe the oxidation rate becomes diffusion controlled at these thicknesses and thus only very thin films are useful as a linear calibration source. Note that the observed oxidation rate continues to decrease very dramatically in the case of the $1 \mu \mathrm{m}$ thick film.

Note that in the interpretation of the QCM data it is important to understand the oxidation process. During the course of our work we realized that the poly-crystalline silver oxidized to the peroxide, $\mathrm{AgO}$, rather than $\mathrm{Ag}_{2} \mathrm{O}$. This was determined by 
measuring the mass uptake of a totally oxidized film with known silver loading, and verified both by heating the film to $200^{\circ} \mathrm{C}$ to form $\mathrm{Ag}_{2} \mathrm{O}$ and by $\mathrm{X}$-ray diffraction analysis of the film.

These limited tests on silver films demonstrate a number of interesting features. From the perspective of a ground test facility, the two that are most important are the demonstrated role of surface contamination and the observation of the time (thickness) variation in the oxidative behavior.

In further studies we examined the temperature dependence of the silver and carbon oxidation rate over the range of $0^{\circ}$ to $85^{\circ} \mathrm{C}$. These results are shown in Figure 4, (For convenience, all results are normalized to a silver reactivity of unity at $273 \mathrm{~K}$ ). Although there is some scatter in the data, the silver oxidation rate is apparently independent of temperature, unlike that for carbon. Thus, we believe that these silver films will provide a good standard for relative investigation of high velocity oxidation behavior of materials. On the other hand, the oxidation rate for this particular form of carbon varies significantly with temperature, a critical effect for predicting lifetime if this material were to be used in an engineering design.

We've also investigated the effect of $\mathrm{O}$-atom velocity on the oxidation rate. This study is complicated by the fact that the number of oxygen atoms per pulse in our beam decreases as the beam velocity increases. Rather than go into the details of our velocity dependent calibration we will only discuss the relative oxidation behavior of the thin silver film and carbon samples as the beam velocity is varied.

The carbon and silver film samples were irradiated simultaneously at velocities of 8,10 and $12 \mathrm{~km} / \mathrm{s}$ at a pulse frequency of $2 \mathrm{~Hz}$. The observed oxidation depth versus time of the thin silver film is shown in Figure 5a. The decreasing oxidation rate with increasing velocity correlates with the decreased number of oxygen atoms produced per pulse as the velocity is increased. Indeed within the uncertainty of our calibration the silver film oxidation efficiency remained constant over the velocity range. (This is not surprising since the oxidation efficiency is near unity.)

This result is contrasted with the observed etched depth histories in the carbon sample as shown in Figure 5b. Here it can be seen that the etch rate increased with velocity even though the $\mathrm{O}$-atom fluence decreased. Thus the carbon sample studied shows a strongly velocity dependent oxidation rate. We have observed a similar trend over the velocity range of 6 to $11 \mathrm{~km} / \mathrm{s}$ in other materials, such as kapton."

Thus oxidative effects on materials can also depend on the velocity of the O-beam employed in the testing. We have shown one instance where different velocity scalings pertain. Assuredly, these will be others. To avoid errors, it is clear that ground testing for lifetime estimates should be performed at the appropriate LEO velocity.

\section{SUMMARY}

Ground testing provides a critical adjunct to the evaluation and validation of material applicability for low earth orbit operations. Care must be taken, however, to ensure that the ground test facility can provide valid test results. In the case of hypersonic oxygen atom testing, proper material evaluation and data interpretation require both a clear understanding of the characteristics of the test facility as well as a standardized measurement protocol.

Details of the PSI fast oxygen atom source have been provided. This system has been used to evaluate materials since 1987, over 2000 material samples have been tested. Good comparison with space test results has been demonstrated. All major beam parameters, e.g., VUV, metastable content, beam velocity spread, have been evaluated and a standard measurement protocol has been applied.

Example test data has been presented for $8 \mathrm{~km} / \mathrm{s}$ oxygen atom beam irradiation of a think silver film and a sample of micro-crystalline carbon. Although these data are of interest in their own right, they also demonstrate the importance of controlling test parameters such as contamination level, sample temperature, and beam velocity.

“Krech, R.H., "Determination of Oxygen Erosion Yield Dependencies Upon Specific LEO Environments," Physical Sciences Inc. TR-1176, March, 1993. 


\section{REFERENCES}

1. Caledonia, G.E., Krech, R.H., and Green, B.D., AlAA J. 25/1, 59 (1987).

2. Leger, L.J., Spiker, I.K., Kuminecz, J.F., and Visentine, J.T., ALAA Paper 83-2631, Oct. 1983.

3. Leger, L.J., Visentine, J.T., and Kuminecz, J.F., AlAA Paper 84-0548, Jan. 1984.

4. Visentine, J.T., Leger, L.J., Kuminecz, J.F., and Spiker, I.K., AIAA paper 85-0415, Jan. 1985.

5. Green, B.D., Caledonia, G.E., and Wilkerson, T.D., J. Spacecraft, 22, 500 (1985).

6. Caledonia, G.E., in Rarefied Gas Dynamics: Space Related Studies, eds. E.P. Muntz, D.P. Weaver and D.H. Campbell, Vol. 116, Progress in Astronautics and Aeronautics, AIAA, Washington, D.C., pp. 129-142, 1989.

7. Levine, A.S., ed., "LDEF - 69 Months in Space, First Post-Retrieval Symposium," NASA Conf. Pub. 3134, 3 vol., June 2-8, 1991.

8. Levine, A.S., ed., "LDEF - 69 Months in Space, Second Post-Retrieval Symposium," NASA Conf. Pub. 3194,4 vol., Second, June 1-5, 1992.

9. Levine, A.S., ed., "LDEF - 69 Months in Space, Third Post-Retrieval Symposium," Abstracts, NASA CP 101 20, Third, Nov. 8-12, 1993.

10. Visentine, J.T., compiler, "Atomic Oxygen Effects Measurements for Shuttle Missions STS-8 and 41-G," 3 vol., NASA TM 100459, Sept. 1988.

11. Leger, L., Koontz, S., Visentine, J., and Hunton, D., "An Overview of the Evaluation of Oxygen Interactions with Materials III Experiment," LDEF Material Results for Spacecraft Applications Conference, Huntsville, AL, Oct. 26-28, 1992. Also AlAA-93-0497, January 1993, Reno, NV.

12. Kamenetzky, R.R., Linton, R.C., Finckenor, M.M. and Vaughn, J.A., "Effects of Atomic Oxygen on Polymeric Materials Flown on EOIM-3) AIAA 93-4103, AIAA Space Programs and Technologies Conference and Exhibit, Sect. 21-23, 1993, Huntsville, AL.

13. Banks, B.A., Aver, B.M., Rutledge, S.K., and deGroh, K.K., "Atomic Oxygen Durability Evaluation of Protected Polymers Using Thermal Energy Plasma Systems," Int. Conf. on Plasma Synthesis and Processing of Materials, Denver, CO, 1993.

14. Koontz, S.L., Albyn, K., and Leger, L.J., J. Spacecraft, 28, 315 (1991).

15. Minton, T.K., "Protocol for Atomic Oxygen Testing of Materials in Ground-Based Facilities, version Number 1," JPL Publication 94-02, April 1, 1994.

16. Caledonia, G.E., Krech, R.H., Upschulte, B.L., Sonnenfroh, D.M., Oakes, D.B., and Holtzclaw, K.W., ALAA Paper 923974, 1992.

17. Caledonia, G.E., Krech, R.H., Upschulte, B.L., Holtzclaw, K.W., and Oakes, D.B., AIAA 94-2638, 1994.

18. Chung, S.Y., Brinza, D.E., Minton, T.K., Stiegman, A.E., Kenny, J.T., and Liang, R.H., "Flight- and Ground-Test Correlation Study of BMDO SDS Materials," JPL Publication 93-31, 1993.

19. Gregory, J.C. and Peters, P.N., "Measurement of the Angular Distribution of 5 eV Oxygen Scattered Off a Solid Surface in Earth Orbit," in Proc. 15th International Symposium on Rarefied Gas Dynamics, Grado, Italy, 1986, eds. V. Boffi and C. Cerignani, pp. 644-656.

20. Oakes, D.B., Krech, R.H., Upschulte, B.L., and Caledonia, G.E., "The Oxidation of Polycrystalline Silver Films by Hyperthermal Oxygen Atoms," submitted to J. Appl. Phys. 
Table 1. Fast O-Beam Properties

\begin{tabular}{|l|l|}
\hline Velocity & $8 \mathrm{~km} / \mathrm{s} \pm 15 \%$ (5 to $12 \mathrm{~km} / \mathrm{s}$ range) \\
Fluence & $\sim 10^{18}$ O-atoms/pulse, $3 \mathrm{~Hz}$ \\
Composition & $>80 \%$ oxygen atoms \\
Size & Expandable to $>1000 \mathrm{~cm}^{2}$ area \\
Charge content & $<1 \%$ ions (controllable by pseudo-Helmholtz coils) \\
Metastable content & O('D) concentration $<0.4 \%$ \\
Temperature & $\mathrm{T} \approx 300 \mathrm{~K}$ \\
VUV/UV content & One photon per $10^{4} \mathrm{O}$ atoms (similar to LEO) \\
\hline
\end{tabular}

Table 2. O-Beam Diagnostics

Velocity, temporal pulse shape

Pulse fluence

Beam composition

Excited state concentrations

Charge content (beam is charge neutral)

Beam shape

Beam translational temperature
Radiometers, mass spectrometer (standard quadrupole and TOF)

Mass flow meter, thin silver film actinometer

Mass spectrometer

Spectrometer, bandpass filters

Langmuir probe

Erosive witness samples, positionable Langmuir probe, 2D visible imaging

Slitted thin silver film target chamber 


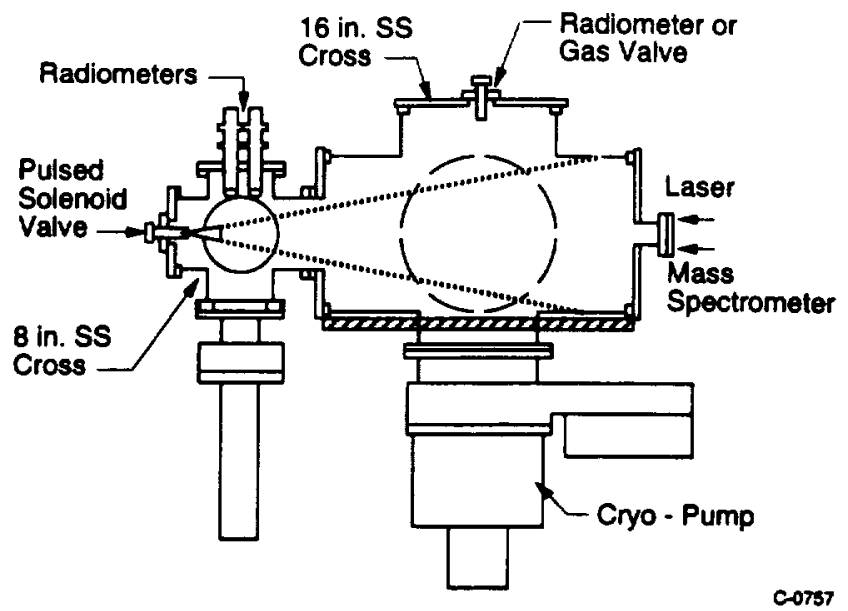

Figure 1. Schematic of the Fast-2 System

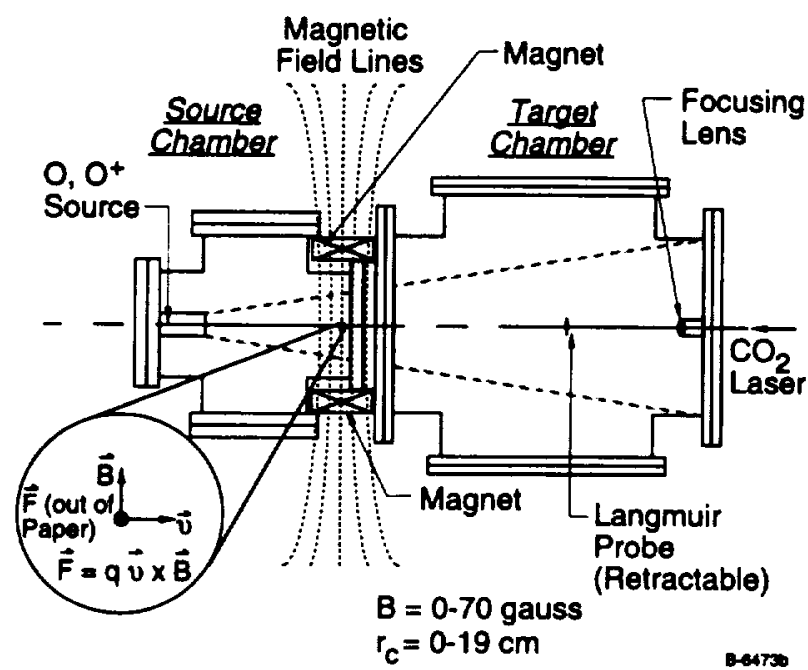

Figure 2. Schematic of beam ion control system.

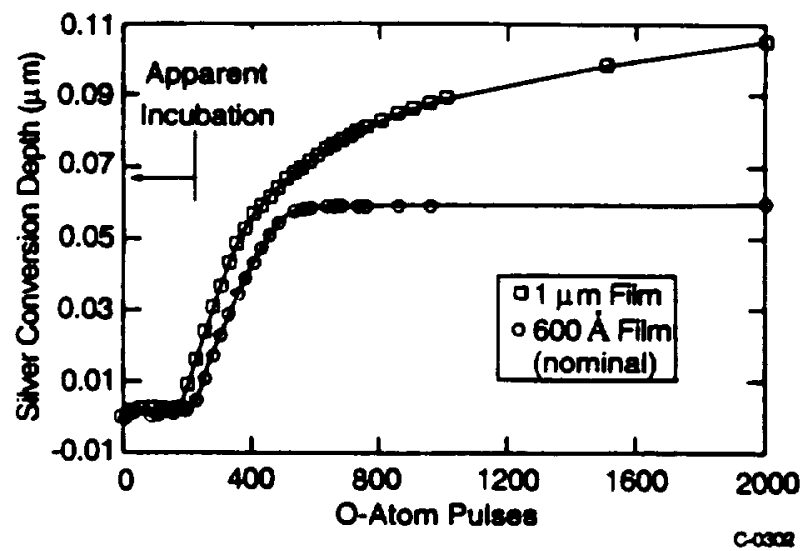

Figure 3. Oxidation behavior of thin silver films coated on QCM crystals (O-atom velocity $=8 \mathrm{~km} / \mathrm{s}$, room temperature). 


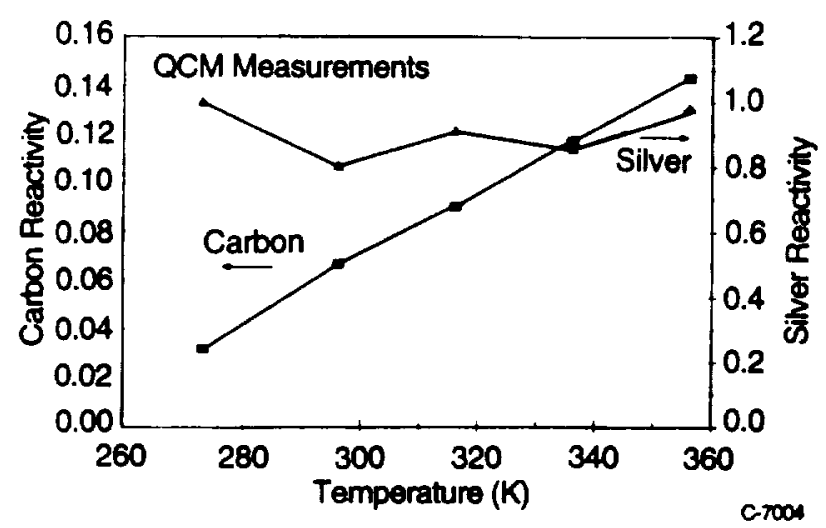

Figure 4. Measured silver and carbon reactivity vs temperature (O-Atom velocity $8 \mathrm{~km} / \mathrm{s})$.

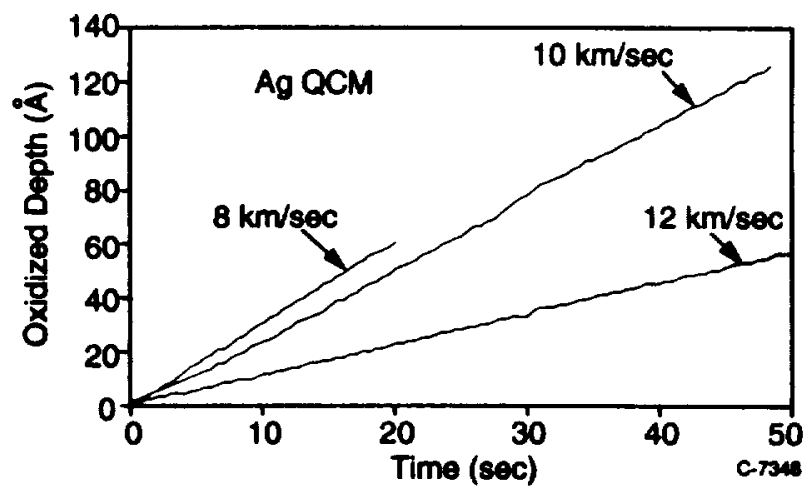

Figure 5a. Observed silver oxidation depth vs time for 3 O-atom velocities. Pulse repetition frequency was $2 \mathrm{~Hz}$.

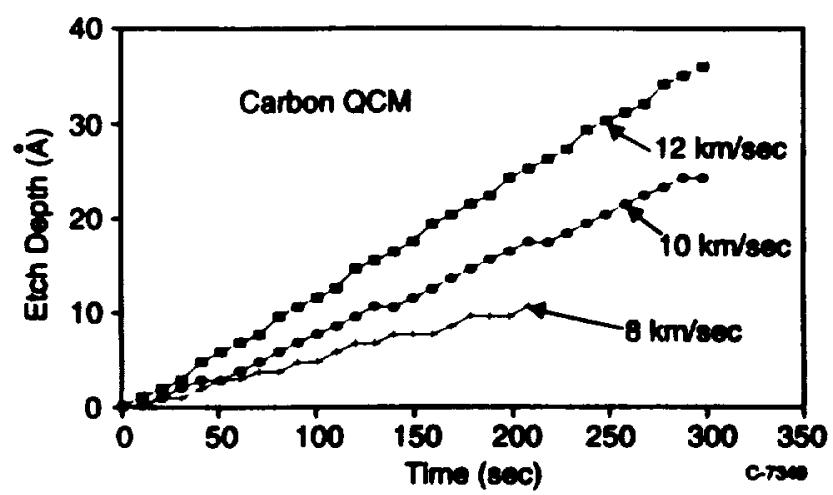

Figure 5b. Observed etch depth of carbon vs time for 30 -atom velocities. Pulse repetition frequency was $2 \mathrm{~Hz}$. 